Network Working Group

Request for Comments: 2698

Category: Informational
J. Heinanen

Telia Finland

R. Guerin

University of Pennsylvania

September 1999

\title{
A Two Rate Three Color Marker
}

Status of this Memo

This memo provides information for the Internet community. It does not specify an Internet standard of any kind. Distribution of this memo is unlimited.

Copyright Notice

Copyright (C) The Internet Society (1999). All Rights Reserved.

Abstract

This document defines a Two Rate Three Color Marker (trTCM), which can be used as a component in a Diffserv traffic conditioner [RFC2475, RFC2474]. The trTCM meters an IP packet stream and marks its packets based on two rates, Peak Information Rate (PIR) and Committed Information Rate (CIR), and their associated burst sizes to be either green, yellow, or red. A packet is marked red if it exceeds the PIR. Otherwise it is marked either yellow or green depending on whether it exceeds or doesn't exceed the CIR.

1. Introduction

The Two Rate Three Color Marker (trTCM) meters an IP packet stream and marks its packets either green, yellow, or red. A packet is marked red if it exceeds the Peak Information Rate (PIR). Otherwise it is marked either yellow or green depending on whether it exceeds or doesn't exceed the Committed Information Rate (CIR). The trTCM is useful, for example, for ingress policing of a service, where a peak rate needs to be enforced separately from a committed rate. 
The Meter meters each packet and passes the packet and the metering result to the Marker:

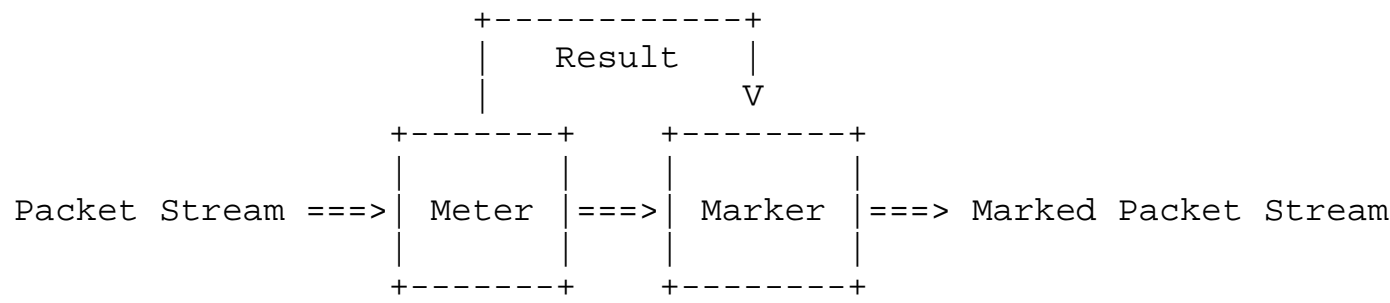

The Meter operates in one of two modes. In the Color-Blind mode, the Meter assumes that the packet stream is uncolored. In the ColorAware mode the Meter assumes that some preceding entity has precolored the incoming packet stream so that each packet is either green, yellow, or red. The details of the pre-coloring process, including handling of error scenarios, and how the Meter determines the color of a pre-colored packet are DS domain specific and outside the scope of this document.

The Marker (re)colors an IP packet according to the results of the Meter. The color is coded in the DS field [RFC2474] of the packet in a PHB specific manner (see section 4 for an example).

A companion document [RFC2697] describes another three color marker, called a Single Rate Three Color Maker (srTCM), where packets are marked based on a single rate and two burst sizes.

2. Configuration

The trTCM is configured by setting its mode and by assigning values to four traffic parameters: a Peak Information Rate (PIR) and its associated Peak Burst Size (PBS) and a Committed Information Rate (CIR) and its associated Committed Burst Size (CBS).

The PIR and CIR are measured in bytes of IP packets per second, i.e., it includes the IP header, but not link specific headers. The PIR must be equal to or greater than the CIR.

The PBS and the CBS and are measured in bytes and both of them must be configured to be greater than 0 . It is recommended that they be configured to be equal to or greater than the size of the largest possible IP packet in the stream. 


\section{Metering}

The behavior of the Meter is specified in terms of its mode and two token buckets, $\mathrm{P}$ and $\mathrm{C}$, with rates $\mathrm{PIR}$ and CIR, respectively. The maximum size of the token bucket $P$ is PBS and the maximum size of the token bucket $\mathrm{C}$ is CBS.

The token buckets $P$ and $C$ are initially (at time 0 ) full, i.e., the token count $\mathrm{TP}(0)=\mathrm{PBS}$ and the token count $\mathrm{TC}(0)=\mathrm{CBS}$. Thereafter, the token count $\mathrm{Tp}$ is incremented by one PIR times per second up to PBS and the token count TC is incremented by one CIR times per second up to CBS.

When a packet of size $B$ bytes arrives at time $t$, the following happens if the trTCM is configured to operate in the Color-Blind mode:

- If $\operatorname{Tp}(t)-B<0$, the packet is red, else

o if $T_{C}(t)-B<0$, the packet is yellow and $T p$ is decremented by $B$, else

o the packet is green and both $\mathrm{TP}$ and $\mathrm{TC}$ are decremented by $\mathrm{B}$.

When a packet of size $B$ bytes arrives at time $t$, the following happens if the trTCM is configured to operate in the Color-Aware mode:

- If the packet has been precolored as red or if $\mathrm{Tp}(t)-B<0$, the packet is red, else

o if the packet has been precolored as yellow or if $\mathrm{TC}(\mathrm{t})-\mathrm{B}<0$, the packet is yellow and $\mathrm{Tp}$ is decremented by $\mathrm{B}$, else

o the packet is green and both $\mathrm{Tp}$ and $\mathrm{Tc}$ are decremented by $\mathrm{B}$.

The actual implementation of a Meter doesn't need to be modeled according to the above formal specification.

4. Marking

The Marker reflects the metering result by setting the DS field of the packet to a particular codepoint. In case of the AF PHB [RFC2597], the color can be coded as the drop precedence of the packet. 
5. Service Example

The trTCM can be used to mark a IP packet stream in a service, where different, decreasing levels of assurances (either absolute or relative) are given to packets which are green, yellow, or red. For example, a service may discard all red packets, because they exceeded the peak rate, forward yellow packets as best effort, and forward green packets with a low drop probability.

6. Security Considerations

The trTCM has no known security concerns.

7. References

[RFC2697] Heinanen, J. and R. Guerin, "A Single Rate Three Color Marker", RFC 2697, September 1999.

[RFC2597] Heinanen, J., Baker, F., Weiss, W. and J. Wroclawski, "Assured Forwarding PHB Group", RFC 2597, June 1999.

[RFC2474] Nichols, K., Blake, S., Baker, F. and D. Black, "Definition of the Differentiated Services Field (DS Field) in the IPv4 and IPv6 Headers", RFC 2474, December 1998 .

[RFC2475] Blake, S., Black, D., Carlson, M., Davies, E., Wang, Z and W. Weiss, "An Architecture for Differentiated Services", RFC 2475, December 1998.

8. Authors' Addresses

Juha Heinanen

Telia Finland, Inc.

Myyrmaentie 2

01600 Vantaa, Finland

EMail: jh@telia.fi

Roch Guerin

University of Pennsylvania

Department of Electrical Engineering, Rm 367 GRW

200 South 33rd Street

Philadelphia, PA 19104

EMail: guerin@ee.upenn.edu 
9. Full Copyright statement

Copyright (C) The Internet Society (1999). All Rights Reserved.

This document and translations of it may be copied and furnished to others, and derivative works that comment on or otherwise explain it or assist in its implementation may be prepared, copied, published and distributed, in whole or in part, without restriction of any kind, provided that the above copyright notice and this paragraph are included on all such copies and derivative works. However, this document itself may not be modified in any way, such as by removing the copyright notice or references to the Internet society or other Internet organizations, except as needed for the purpose of developing Internet standards in which case the procedures for copyrights defined in the Internet Standards process must be followed, or as required to translate it into languages other than English.

The limited permissions granted above are perpetual and will not be revoked by the Internet society or its successors or assigns.

This document and the information contained herein is provided on an "AS IS" basis and THE INTERNET SOCIETY AND THE INTERNET ENGINEERING TASK FORCE DISCLAIMS ALL WARRANTIES, EXPRESS OR IMPLIED, INCLUDING BUT NOT LIMITED TO ANY WARRANTY THAT THE USE OF THE INFORMATION HEREIN WILL NOT INFRINGE ANY RIGHTS OR ANY IMPLIED WARRANTIES OF MERCHANTABILITY OR FITNESS FOR A PARTICULAR PURPOSE.

Acknowledgement

Funding for the RFC Editor function is currently provided by the Internet society. 